\title{
Influência da governança pública na eficiência da alocação dos
} recursos públicos

Rodolfo Rocha dos Santos ${ }^{1}$

Suliani Rover ${ }^{2}$

1 Universidade Federal Rural do Rio de Janeiro / Departamento de Ciências Contábeis e Finanças, Seropédica / RJ — Brasil

${ }^{2}$ Universidade Federal de Santa Catarina / Programa de Pós-Graduação em Contabilidade, Florianópolis / SC — Brasil

Este estudo buscou verificar a influência de práticas de governança pública na eficiência da aplicação dos recursos públicos em educação e saúde nos municípios brasileiros, a partir da perspectiva da nova governança pública (new public governance - NPG). Para atingir tal objetivo, construiu-se um índice de eficiência municipal (IEM) com o intuito de medir a eficiência da aplicação dos recursos públicos com educação e saúde dos municípios brasileiros no ano de 2010. Posteriormente, por meio de modelos estatísticos de regressões de Mínimos Quadrados Ordinários, Tobit bootstraping e quantílica, verificou-se a influência de variáveis tidas como princípios de governança pública: transparência/accountability, participação, integridade/ética, conformidade legal, equidade e efetividade. Como conclusão, este artigo mostra que práticas de governança pública influenciam a eficiência da aplicação dos recursos públicos em educação e saúde nos municípios brasileiros.

Palavras-chave: governança pública; gestão pública; eficiência; avaliação de desempenho; gastos públicos.

\section{Influencia de la gobernanza pública en la eficiencia de la asignación de los recursos públicos}

Este estudio buscó verificar la influencia de prácticas de gobernanza pública en la eficiencia de la aplicación de los recursos públicos en educación y salud en los municipios brasileños, desde la perspectiva de la nueva gobernanza pública (NPG). Para alcanzar tal objetivo, se construyó un índice de eficiencia municipal (IEM) con la intención de medir la eficiencia de la aplicación de los recursos públicos en educación y salud en los municipios brasileños en el año 2010. Posteriormente, por medio de modelos estadísticos de regresiones de Mínimos Cuadrados Ordinarios, Tobit bootstraping y cuantílica, se verificó la influencia de variables consideradas como principios de gobernanza pública: transparencia/accountability, participación, integridad/ética, cumplimiento legal, equidad y efectividad. Como conclusión, este artículo muestra que las prácticas de gobernanza pública influencian la eficiencia de la aplicación de los recursos públicos en educación y salud en los municipios brasileños.

Palabras clave: gobernanza pública; eficiencia; educación; salud; gastos públicos.

\section{Influence of public governance on the efficiency in the allocation of public resources}

This study sought to verify the influence of public governance practices on the efficiency in the application of public resources in education and health in Brazilian municipalities, based on the perspective of the new public governance (NPG). A municipal efficiency index (MEI) to measure the efficiency in the allocation of public resources in education and health in Brazilian municipalities in 2010. Then, using the statistical models of Ordinary Least Squares, bootstrapped Tobit regression, and quantile regression, the influence of variables considered principles of public governance: transparency/accountability, participation, integrity/ethics, legal conformity, equity, and effectiveness. In conclusion, the study shows that public governance practices influence the efficiency in the application of public resources in education and health in Brazilian municipalities.

Keywords: public governance; efficiency; public administration; performance evaluation; public spending. 


\section{INTRODUÇÃO}

Recentes fenômenos, como a crise econômica e financeira mundial, exigem principalmente que o Estado se reinvente para melhorar seu desempenho quanto ao cumprimento de sua função social (Matias-Pereira, 2010). O mesmo autor destaca a crise do Welfare State nos países desenvolvidos, a crise do desenvolvimentismo para os países em desenvolvimento, o esgotamento do modelo econômico, a fragilidade do modelo político e a deficiência do modelo administrativo como catalizadores dessa mudança.

Nesse novo cenário, que vem sendo construído ao longo dos últimos trinta anos, o Estado passa a reafirmar seu papel de apoio ao mercado, por medir, regular, estimular e promover intervenções e regulações, o que exige dos governantes e administradores públicos a adoção de ações inovadoras na gestão do setor público (Matias-Pereira, 2010). A relação entre o Estado e a sociedade passa a ser vista sob outra perspectiva: o Estado deixa de desempenhar somente o papel de governo e adota um papel de governança (Kissler \& Heidemann, 2006).

Kormendi e Meguire (1985) foram os primeiros a relacionar práticas de governança pública com crescimento econômico de uma nação. Os autores introduziram uma medida de liberdades civis em seu conjunto de variáveis e observaram correlação positiva com o crescimento econômico. Desde então, outros estudos surgiram nesse cenário (Asatryan \& Witte, 2015; Chang, 2014; A. G. Oliveira \& Pisa, 2015). No entanto, percebe-se um entendimento mais macroeconômico acerca desse fenômeno, o que desperta o interesse de entender, no nível microeconômico, se esse fenômeno também é percebido.

Dentro desse escopo, entender a eficiência da alocação dos recursos públicos no que diz respeito à educação e saúde, nesta pesquisa, apresenta-se como preocupação microeconômica, que se mostra com diversos entraves para seu desenvolvimento. Um dos problemas comumente debatidos pela academia é a falta de eficiência na utilização dos recursos disponíveis para o desenvolvimento socioeconômico.

Peña (2008) explica que eficiência é a combinação ótima dos insumos e métodos necessários (inputs) no processo produtivo, de modo que resulte no máximo de produtos possíveis (outputs). Isto é, eficiência é a capacidade de fazer corretamente as ações, de minimizar a relação entre insumo e produto e, desse modo, otimizar a utilização de recursos. Mello, Angulo-Meza, Gomes, Fernandes e Biondi (2008) afirmam que eficiência consiste em comparar o que foi produzido, dados os recursos disponíveis, com o que poderia ter sido produzido com os mesmos recursos.

Dentre as diversas metodologias que permitem avaliar a eficiência, Peña (2008) evidencia que o método de análise envoltória de dados (data envelopment analysis - DEA) tem sido aplicado com sucesso em estudos de eficiência da administração pública.

O problema de pesquisa que este estudo se propôs a investigar gira em torno do fato de que a não adoção de medidas de governança pública por parte das instituições governamentais podem diminuir a eficiência na aplicação dos seus recursos, o que proporciona menor benefício à sociedade e menor crescimento da economia. Diante disso, o desconhecimento da influência das práticas de governança pública na gestão municipal pode reduzir o bem-estar social proporcionado pelas funções do Estado. Para nortear a análise desse problema, formula-se a seguinte questão de pesquisa:

- Qual é a influência das práticas de governança pública na eficiência da aplicação dos recursos públicos em educação e saúde dos municípios brasileiros? 
Então, tem-se por objetivo verificar a influência de práticas de governança pública na eficiência da alocação dos recursos públicos em educação e saúde dos municípios brasileiros.

Para tanto, construiu-se um modelo de DEA com retornos variáveis de escala (variable return scale - VRS) com orientação output para mensurar a eficiência da aplicação dos recursos públicos em educação e saúde dos municípios brasileiros para o ano de 2010, e o índice de eficiência municipal (IEM) confome Costa, Ferreira, Braga e Abrantes (2015). Posteriormente, aplicaram-se os modelos de regressão de mínimos quadrados ordinários (MQO), Tobit com bootstrapping e regressão quantílica para verificar a influência de variáveis de governança pública nas escalas de eficiência construídas e no IEM - variáveis estas baseadas nos princípios de governança pública conforme Pisa (2014).

Para atingir o objetivo proposto, este artigo se divide em cinco seções, incluindo esta introdução, que contextualiza o tema práticas de governança pública. A segunda seção elucida o movimento de governança pública, suas conceitualizações e seus princípios, além de apresentar a eficiência da alocação dos recursos. A terceira seção apresenta os modelos de escala de eficiência construídos a partir da DEA, o IEM e os modelos estatísticos para verificar a influência de práticas de governança pública na eficiência da aplicação dos recursos públicos em educação e saúde. A seção quatro apresenta os resultados obtidos e a quinta seção traz a conclusão e as sugestões para futuras pesquisas.

\section{FUNDAMENTAÇÃO TEÓRICA}

\subsection{Movimento de governança pública}

Enquanto alguns estudiosos e profissionais seguem ideais neoliberais e defendem a aplicação de princípios de organizações privadas na gestão pública, com seus movimentos gerencialistas, outros acreditam na concepção de administração pública como instrumento de um projeto democratizante (Dias \& Cario, 2014a; Kickert, 1997; Pedersen \& Johannsen, 2018). Dessa maneira, concomitantemente com o surgimento da nova gestão pública (new public management - NPM) e outros movimentos gerencialistas, também surgiu um movimento da administração pública conhecido como governança pública, que vem sendo debatido em uma abordagem multidisciplinar (Dias \& Cario, 2014a; Lynn \& Malinowska, 2018).

Movimentos sociais argumentam que a eficiência e abrangência dos serviços do setor público podem reviver o éthos da prática de governança para estimular o interesse público (Mandeli, 2016). Isso pode ser alcançado por meio da promoção de redes comunitárias e pode encorajar a colaboração multiagentes. Ambos os argumentos podem ser considerados o principal impulso para a reforma do setor público e podem ser vistos como fundamentos teóricos da nova governança pública (new public governance - NPG) (Considine \& Lewis, 2003).

Comparando a governança pública com a NPM, alguns autores usam os termos como sinônimos para ilustrar mudanças que impulsionaram reformas gerenciais e rotinas de administração pública “ortodoxas", como Hood (1991). Entretanto, a maioria dos estudiosos (Dias \& Cario, 2014b; Kickert, 1997; Kissler \& Heidemann, 2006; Mandeli, 2016; Peters \& Pierre, 1998) argumentam que eles são diferentes.

Secchi (2009, p. 358) utilizou a proeminente definição das ciências políticas de administração pública: "um modelo horizontal de relação entre atores públicos e privados no processo de elaboração de políticas públicas”. 
Para Dias e Cario (2014b), governança pública passa a ser mais do que um simples artifício da administração pública, trata-se de um movimento de reforma da gestão pública. Os autores definem governança pública como

[...] uma estratégia desenvolvimentista adotada na relação entre o Estado e a sociedade com vistas à construção do público, envolvendo o próprio público e buscando atender [a] os interesses desse mesmo público. Em outras palavras, uma governança que se utiliza de processos mais democráticos para desenhar as soluções demandadas pela sociedade do século XXI (Dias \& Cario, 2014b, p. 93).

Kickert (1997) define governança pública como a gestão de redes complexas, consistindo em muitos atores diferentes do governo nacional, provincial e local, grupos políticos e sociais, grupos de pressão, grupos de ação e interesse, instituições sociais, organizações privadas e outras mais. A gestão de tais redes públicas é uma forma de controle externo do governo. Governança pública significa influenciar processos sociais em uma rede de políticas públicas de muitos outros atores.

De acordo com Lynn e Malinowska (2018), a governança pública é um campo de estudo interdisciplinar centrado nas relações de poder entre as autoridades governamentais, a sociedade civil e o mercado, em um contexto de transformações na capacidade das comunidades políticas legitimamente se governarem e agirem efetivamente.

Por fim, Pedersen e Johannsen (2018) evidenciam que a NPG enfatiza que serviços mais eficientes e melhores resultados podem ser alcançados por meio de processos administrativos flexíveis nos quais a confiança, a inclusão de cidadãos e o aprimoramento dos contatos relacionais público-privado funcionam como mecanismo central da governança.

\subsection{Princípios da governança pública}

De acordo com o Banco Mundial, para que a governança pública seja efetiva, presume-se a presença de um Estado de direito, de uma sociedade civil participativa em relação aos assuntos públicos, de uma burocracia insuflada de ética profissional, de políticas planejadas de modo previsível, aberto e transparente e de um braço executivo que se responsabilize por suas ações (World Bank, 2007; Tribunal de Contas da União [TCU], 2014).

O Banco Mundial tem como princípios de boa governança a legitimidade, a equidade, a responsabilidade, a eficiência, a probidade, a transparência e a accountability (Pisa, 2014).

Pisa (2014) destaca que existe uma recorrente preocupação com a transparência, a accountability, a integridade e a participação quando se discute princípios de governança pública. Para Matias-Pereira (2010) são quatro os princípios de governança pública: a) relações éticas; b) conformidade em todas as dimensões; c) transparência; e d) accountability. $\mathrm{O}$ autor também assevera que, para existir a boa governança, deve estar presente o fator "participação proativa de todos os atores envolvidos, dirigentes, políticos, órgãos de controle e, em especial, da sociedade organizada" (Matias-Pereira, 2010, p. 124).

Pisa (2014) utiliza os seguintes princípios de governança pública: a) transparência; b) accountability; c) participação; d) integridade/ética; e) conformidade legal; f) equidade; e g) efetividade. A autora destaca que é quase unânime na literatura a citação dos princípios da transparência, accountability, integridade e participação. O Quadro 1 ilustra a conceitualização de cada princípio de governança pública apresentada por Pisa (2014). 


\section{QUADRO 1 PRINCÍPIOS DE GOVERNANÇA PÚBLICA}

\begin{tabular}{|c|c|}
\hline Princípio & Conceito \\
\hline Transparência & $\begin{array}{l}\text { No exercício da governança, "fornecer aos cidadãos mais informações e acesso ao governo e seus } \\
\text { processos de tomada de decisão contribui significativamente para uma maior abertura dentro da } \\
\text { administração" (Organisation for Economic Co-Operation and Development [OECD], 2001, p. 73). }\end{array}$ \\
\hline Accountability & $\begin{array}{l}\text { Refere-se à obrigatoriedade do gestor público de prestar contas de suas decisões e ações à } \\
\text { sociedade que lhe delegou o poder para tal, o que necessariamente implica reprimenda aos } \\
\text { comportamentos que apresentarem desvios de conduta (Pisa, 2014). }\end{array}$ \\
\hline Participação & $\begin{array}{l}\text { A qualidade, pertinência e eficácia das políticas dependem da ampla participação em toda a } \\
\text { cadeia política, desde a concepção até a implementação. } 0 \text { respaldo da participação promove } \\
\text { a confiança, tanto no alcance do resultado final como nas instituições que criam as políticas } \\
\text { (European Commission, 2001). }\end{array}$ \\
\hline Integridade/ética & $\begin{array}{l}\text { "Baseia-se na honestidade e na objetividade, além de elevados padrões de propriedade e } \\
\text { probidade na administração dos recursos públicos e privados e na gestão dos assuntos de uma } \\
\text { entidade. Depende da eficácia do quadro de controle e dos padrões pessoais e do profissionalismo } \\
\text { dos indivíduos dentro da entidade. Reflete-se tanto nos procedimentos de tomada de decisão } \\
\text { da entidade quanto na qualidade de seus relatórios financeiros e de desempenho (International } \\
\text { Federation of Accountants" [IFAC], 2001, p.12). }\end{array}$ \\
\hline Conformidade legal & $\begin{array}{l}\text { Diz respeito ao cumprimento das leis e regulamentos prescritos para a administração pública, } \\
\text { além de atender ao princípio da legalidade, o gestor deve fazê-lo com eficiência e eficácia, } \\
\text { atendendo ao interesse público, ou seja, com efetividade (Pisa, 2014). }\end{array}$ \\
\hline Equidade & $\begin{array}{l}0 \text { conceito de equidade está intimamente ligado ao conceito de justiça e pressupõe o tratamento } \\
\text { desigual aos desiguais na busca pela igualdade, em um claro reconhecimento das desigualdades } \\
\text { geradas na pólis (Aristóteles, 2002). }\end{array}$ \\
\hline Efetividade & $\begin{array}{l}\text { Refere-se aos impactos gerados pelos produtos/serviços, processos ou projetos. A efetividade } \\
\text { está vinculada ao grau de satisfação ou, ainda, ao valor agregado (Pisa, 2014). }\end{array}$ \\
\hline
\end{tabular}

Fonte: Adaptado de Pisa (2014).

Assim, este estudo utilizou os princípios de governança pública descritos anterioremente e apresentados por A. G. Oliveira e Pisa (2015). Vale ressaltar que os autores exploram o conceito de efetividade no índice de governança pública que constroem. Entretanto, recorreu-se às variáveis de governança pública tidas por A. G. Oliveira e Pisa (2015) como indicadores na construção do índice de avaliação de governança pública (IGovP), em função de cada princípio adotado, para verificar a eficiência na aplicação dos recursos públicos em educação e saúde em relação a tais variáveis. Entende-se que a busca por uma aplicação eficiente dos recursos públicos está inserida nos objetivos da administração pública, a fim de se atingir a efetividade das políticas públicas (I. G. S. Oliveira, 2015). A. G. Oliveira e Pisa (2015, p. 1271) afirmam, ainda, que:

[...] para desenvolver indicadores de governança é importante levar em conta alguns critérios de análise, como: [...] a eficiência, na medida em que as políticas reflitam o adequado retorno social em função da utilização de recursos escassos. 


\subsection{Eficiência da alocação dos recursos públicos}

De acordo com S. G.Chan e Karim (2012, p. 8):

Eficiência da despesa pública é definida como a capacidade do governo para maximizar suas atividades econômicas, dado um nível de gastos, ou a capacidade do governo para minimizar seus gastos, dado um nível de atividade econômica. Eficiência da despesa pública é definida como a capacidade do governo para maximizar suas atividades econômicas, dado um nível de gastos, ou a capacidade do governo para minimizar seus gastos, dado um nível de atividade econômica.

O desempenho do setor público reflete o desempenho acumulado dos seguintes setores e resultados governamentais: administração, educação, saúde, distribuição de renda, estabilidade de renda e desempenho econômico (Baciu \& Botezat, 2014). Todos são indicadores normalmente considerados na literatura como benchmarks do desempenho do setor público (Afonso, Schuknecht, \& Tanzi, 2010).

Tem-se em vista a importância dos investimentos realizados nos setores educação e saúde para o crescimento econômico de uma sociedade e a diminuição de discrepâncias sociais, tanto em termos de bem-estar como de renda, completando a ideia de desenvolvimento paraeconômico trazida por Guerreiro Ramos (1989). Este estudo visou a se ligar a tais funções governamentais.

Na perspectiva dos setores estudados nesta pesquisa, Araújo-Júnior, Justo, Rocha e Gomes (2017) discutem que a educação é o caminho mais oportuno para promover o desenvolvimento de um país. A Organização das Nações Unidas para a Educação, a Ciência e a Cultura (Unesco, 1998) tem a educação básica como pilar que estrutura a formação do capital humano de um país.

De acordo com Lopes, Toyoshima e Gomes (2009), nos países em desenvolvimento as conjunções de saúde pública são acentuadas por conta das péssimas condições socioeconômicas vivenciadas pela maioria da população. Os autores expõem, ainda, que por conta disso, o sistema público de saúde assume vital importância no sentido de reduzir os problemas da população menos favorecida, mais vulnerável às doenças (Lopes et al., 2009).

Desse modo, a ineficiência na aplicação dos recursos públicos nos setores educação e saúde só agrava a desigualdade social e prejudica o crescimento econômico de um país.

Nesse sentido, Asatryan e Witte (2015) estudaram o papel da democracia direta e participativa na provisão eficiente de bens públicos por parte dos municípios do estado alemão da Bavária. Os autores constataram que práticas democráticas mais diretas estão associadas com maior eficiência do governo na provisão de bens e serviços, o que sugere que as práticas de governança pública surtem efeitos positivos na eficiência do Estado.

Chang (2014) verificou a relação entre a governança pública e a eficiência de empresas geradoras de energia elétrica da China e de Taiwan. Demonstrou-se que a boa qualidade da governança pública elevou a eficiência da indústria de energia elétrica chinesa.

No Brasil, J. L. M. Silva e Almeida (2012) fizeram uso da DEA para construir uma escala de eficiência dos municípios do Rio Grande do Norte quanto aos gastos públicos com educação e, por meio do modelo de regressão Tobit, verificaram o impacto de diversas variáveis na ineficiência atingida pelas decision-making units (DMU), dentre elas a presença de conselhos municipais de educação. Os autores observaram uma relação negativa entre a ineficiência dos municípios e a variável em questão, o que sugere o incremento da eficiência da máquina pública a partir de práticas de governança. 


\section{PROCEDIMENTOS METODOLÓGICOS}

\subsection{Análise envoltória de dados (data envelopment analysis)}

Existem inúmeras metodologias para medir a eficiência. Dentre todas elas, Lampe e Hilgers (2015) evidenciam que a mais utilizada é a DEA. Na administração pública, diversos estudos recorreram à DEA para mensuração da eficiência, como J. L. M. Silva e Almeida (2012), Sibiano e Agasisti (2013), Costa et al. (2015), Samut e Cafri (2016), entre outros.

Esta pesquisa adotou o modelo DEA-VRS. Optou-se pela orientação output, que mede quanto a produção pode ser expandida sem que sejam alterados os inputs - como expressa a seguinte equação:

$$
\begin{aligned}
& \operatorname{Max} E_{c}=\sum_{j=1}^{s} u r+y_{r o}+u_{*} \\
& \text { S. a.: } \sum_{i=1}^{m} v_{i} x_{i c}=1 \\
& \sum_{j=1}^{s} u_{r} y_{r j}+u_{*}-\sum_{i=1}^{m} v_{i} x_{i o} \leq 0, \forall j \\
& u_{r} \geq 0, v_{i} \geq 0, \forall r, i \\
& u_{*} \in \mathfrak{R}
\end{aligned}
$$

Também se optou pela aplicação do modelo BCC - proposto por Banker, Charnes e Cooper (1984), de acordo com o que é recomendado por Souza e Gasparini (2006), com o intuito de considerar a disparidade de tamanho existente entre as decision-making units (DMU).

\subsection{Universo de pesquisa}

O universo de pesquisa consistiu nos municípios brasileiros, no ano de 2010, que apresentaram dados suficientes para a construção das escalas de eficiência. O Brasil tinha 5.565 municípios em 2010. Foram excluídos da amostra os municípios que não apresentaram informação em ao menos uma variável ou aqueles que apresentaram alguma variável com valor acima de 3 desvios padrão no teste padronizado (Z-teste) ${ }^{1}$, conforme Diniz (2012). Desse modo, a amostra final contemplou, ao todo, 3.193 municípios brasileiros. A amostra representa 57,37\% dos municípios brasileiros no ano de 2010 .

Optou-se pelo ano de 2010 por ter sido o último no qual se realizou o censo nacional. Faria, Jannuzzi e Silva (2008) justificam que, dada a rigidez do orçamento público, o nível identificado de aplicação dos recursos públicos em educação e saúde, por volta de um ano, é representativo para alguns dos anos seguintes.

\footnotetext{
${ }^{1} \mathrm{O}$ teste do escore padronizado é dado pela seguinte equação:

$Z_{i}=\frac{x i-x}{S}$

Onde:

$x$ é a média amostral

S é o desvio padrão amostral
} 


\subsection{Construção da escala de eficiência e o índice de eficiência municipal}

O Quadro 2 apresenta as variáveis utilizadas para medir a eficiência da aplicação dos recursos públicos na educação dos municípios brasileiros.

\section{QUADRO 2 VARIÁVEIS UTILIZADAS COMO INPUTS E OUTPUTS PARA O SETOR EDUCAÇÃO}

\begin{tabular}{|c|c|}
\hline Inputs & Descrição \\
\hline GpaEdu & $\begin{array}{l}\text { Gasto por aluno com educação: gastos municipais no setor educação divididos } \\
\text { pelo total de alunos do Ensino Infantil e do Ensino Fundamental. }\end{array}$ \\
\hline Valor adicionado fiscal per capita & Produto interno bruto municipal (PIBM) per capita. \\
\hline Outputs & Descrição \\
\hline IDEB $5^{\circ}$ ano & $\begin{array}{l}\text { Índice de desenvolvimento da educação básica (IDEB) dos alunos do } 5^{\circ} \text { ano do } \\
\text { Ensino Fundamental. }\end{array}$ \\
\hline IDEB $9^{\circ}$ ano & IDEB dos alunos do $9^{\circ}$ ano do Ensino Fundamental. \\
\hline InTxAtr\% & $\begin{array}{l}\text { Inverso da taxa de alunos de } 6 \text { a } 14 \text { anos no Ensino Fundamental com } 2 \text { anos } \\
\text { ou mais de atraso. }\end{array}$ \\
\hline TaxAt6a14\% & Taxa de atendimento a crianças de 6 a 14 anos na escola. \\
\hline
\end{tabular}

Fonte: Elaborado pelos autores.

A variável GpaEdu considerou todos os gastos com educação de responsabilidade do município ponderados pelo total de alunos do Ensino Infantil e do Ensino Fundamental de cada um. Busca-se, assim, mensurar a alocação dos recursos públicos com educação.

O valor adicionado fiscal per capita é mensurado pelo produto interno bruto municipal (PIBM) ponderado pela população total do município. A introdução dessa variável visa a captar os efeitos que podem ser gerados pela riqueza municipal sobre a eficiência da aplicação de seus recursos públicos. A. A. P. Silva, Ferreira, Braga e Abrantes (2012) e Costa et al. (2015) fazem uso desse artifício.

Quanto aos outputs, buscaram-se variáveis que compreendessem os 3 componentes apresentados por Araújo, Conde e Luzio (2007): a) componente desempenho educacional; b) componente adequação idade-série; e c) componente atendimento escolar.

Para construir a escala de eficiência da aplicação dos recursos públicos com o setor saúde foram selecionadas as variáveis apresentadas no Quadro 3.

A variável GpcSau\&S engloba os gastos com saúde e saneamento ponderados pela população total do município em questão. Os gastos com saneamento são considerados, aqui, por conta de existir uma relação entre a cobertura de abastecimento de água e esgoto e a redução da mortalidade infantil (K. E. A. Cruz \& Ramos, 2012). 


\section{QUADRO 3 VARIÁVEIS UTILIZADAS COMO INPUTS E OUTPUTS PARA 0 SETOR SAÚDE}

\begin{tabular}{|c|c|c|}
\hline Inputs & Descrição & Fonte \\
\hline GpcSau\&S & $\begin{array}{l}\text { Gasto per capita com saúde e saneamento: } \\
\text { gastos municipais no setor saúde e saneamento. }\end{array}$ & Secretaria do Tesouro Nacional (STN). \\
\hline $\begin{array}{l}\text { Valor adicionado fiscal } \\
\text { per capita }\end{array}$ & Produto interno bruto municipal (PIBM) per capita. & $\begin{array}{l}\text { Instituto Brasileiro de Geografia e Estatística } \\
\text { (IBGE). }\end{array}$ \\
\hline Outputs & Descrição & \\
\hline PaPSF\% & $\begin{array}{l}\text { Percentual da população atendida pelo Programa } \\
\text { Saúde da Família (PSF). }\end{array}$ & Ministério da Saúde. \\
\hline InTxMorlnf\% & Inverso da taxa de mortalidade infantil. & IBGE. \\
\hline NasVivM7cpn\% & $\begin{array}{l}\text { Percentual de nascidos vivos cujas mães } \\
\text { realizaram } 7 \text { ou mais consultas de pré-natal. }\end{array}$ & $\begin{array}{l}\text { Pesquisa Nacional por Amostra de Domicílios } \\
\text { (PNAD). }\end{array}$ \\
\hline DParPercSan\% & $\begin{array}{l}\text { Proporção de domicilios particulares } \\
\text { permanentes com saneamento adequado. }\end{array}$ & PNAD. \\
\hline
\end{tabular}

Fonte: Elaborado pelos autores.

Do mesmo modo que o modelo voltado à aplicação dos recursos públicos com educação, no modelo voltado à saúde considerou o produto interno bruto (PIB) per capita uma variável não discricionária.

Em relação aos outputs, buscou-se utilizar aqueles relacionados ao desenvolvimento e à proteção da saúde e aos serviços de tratamento e acompanhamento de questões voltadas à saúde. Por haver estruturas bem diferenciadas dentre os municípios brasileiros e seus sistemas de saúde, além de existirem diversas ações de competência compartilhada, optou-se por comparar o desempenho dos municípios quanto aos resultados diretos voltados à atenção básica de saúde - responsabilidade exclusiva de cada município (Varela, Martins, \& Fávero, 2012).

Utilizou-se o software Open Source DEA para gerar as escalas de eficiência da aplicação dos recursos públicos em educação e saúde.

Depois das escalas de eficiência para os setores educação e saúde propostas por esta pesquisa, construiu-se uma adaptação do IEM proposto por Costa et al. (2015).

Esta pesquisa utiliza apenas os setores educação e saúde, conforme a seguinte equação:

$$
I M E_{i j}=\theta_{\text {edu }} \times \frac{G e d u}{\text { Gtotal }}+\theta_{\text {sau }} \times \frac{\text { Gsau }}{\text { Gtotal }}
$$

onde IEM representa o índice de eficiência municipal para o i-ésimo município da amostra para o ano j, $\theta$ é o escore de eficiência relativa obtido pela DEA nos setores educação e saúde, no qual se multiplica pela razão entre os gastos em cada dimensão analisada e o gasto total do município (Costa et al., 2015). 
Buscou-se construir o IEM com o intuito de analisar em um único índice a eficiência da aplicação dos recursos públicos tanto no setor saúde quanto no setor educação.

\subsection{PROCEDIMENTOS DE ANÁLISE DE DADO}

\subsubsection{Modelos adotados}

Segundo Hoff (2007), tanto o modelo de regressão Tobit quanto o modelo de mínomos quadrados ordinários (MQO), são suficientes para o chamado "segundo estágio" da DEA. Porém, Adkins (2014) afirma que o modelo de regressão Tobit é mais adequado para a segunda fase da DEA, pois o MQO acaba tornando-se tendencioso pela característica truncada, ou censurada, da eficiência.

Diniz (2012) argumenta que a utilização do método Tobit não apresenta fundamentação consistente. Simar (2003) evidenciou que os intervalos do Tobit se apresentam longe dos valores de $\beta$ reais estimados por bootstrapping.

O Tobit com bootstrapping é utilizado para que se tenha maior robustez nos métodos não paramétricos (Diniz, 2012). Ao obter uma mesma fronteira, inúmeras vezes, por bootstrapping, retira-se a correlação existente dos valores de $\beta$, eliminando inclusive a parte aleatória do erro que pode recorrer em uma ineficiência (Diniz, 2012).

De acordo com Costa et al. (2015), esses modelos de análise censurada não consideram o caso no qual as variáveis relacionadas ao sistema de produção podem não influenciar igualmente as unidades observadas. Desse modo, Costa et al. (2015) observam que esse problema pode ser contornado ao fazer uso da regressão quantílica, com a finalidade de verificar as diferenças na associação entre as variáveis estudadas nos quantis da distribuição.

Este estudo aplicou um modelo MQO, um modelo de regressão Tobit com bootstrapping e um modelo de regressão quantílica.

\subsubsection{Variáveis explicativas}

A fim de verificar a influência de práticas de governança pública na eficiência da aplicação dos recursos públicos em educação e saúde, selecionaram-se variáveis que representam indicadores aqui associados aos princípios de governança pública apresentados anteriormente.

O Quadro 4 apresenta os princípios de governança pública, as variáveis utilizadas como proxies para mensurá-los e a fonte dos dados.

\section{QUADRO 4 VARIÁVEIS DE GOVERNANÇA PÚBLICA}

\begin{tabular}{|c|c|c|c|}
\hline Princípio & Variável & Fonte & $\begin{array}{l}\text { Relação } \\
\text { esperada }\end{array}$ \\
\hline $\begin{array}{l}\text { Transparência e } \\
\text { accountability }\end{array}$ & $\begin{array}{l}\text { Índice de responsabilidade fiscal, social e gestão } \\
\text { (IRFS). }\end{array}$ & $\begin{array}{l}\text { Confederação Nacional de Municípios } \\
\text { (CNM). }\end{array}$ & Positiva \\
\hline
\end{tabular}




\begin{tabular}{|c|c|c|c|}
\hline Princípio & Variável & Fonte & $\begin{array}{l}\text { Relação } \\
\text { esperada }\end{array}$ \\
\hline \multirow{4}{*}{ Participação } & $\begin{array}{l}\text { Percentual de eleitores: votos válidos versus eleitores } \\
\text { aptos — percentual no } 1^{\circ} \text { turno (EIV/EIA\%). }\end{array}$ & Tribunal Superior Eleitoral (TSE). & Positiva \\
\hline & $\begin{array}{l}\text { Taxa de participação em Conselhos Nacionais e } \\
\text { Municipais (TxParCM). }\end{array}$ & $\begin{array}{l}\text { Instituto Brasileiro de Geografia e } \\
\text { Estatística (IBGE). }\end{array}$ & Positiva \\
\hline & $\begin{array}{l}\text { Presença de Conselho Municipal de Educação } \\
\text { (PresCME). }\end{array}$ & $\begin{array}{l}\text { Site da União Nacional dos Conselhos } \\
\text { Municipais de Educação. }\end{array}$ & Positiva \\
\hline & $\begin{array}{l}\text { Presença de Conselho Municipal de Saúde } \\
\text { (PresCMS). }\end{array}$ & $\begin{array}{l}\text { Pesquisa Nacional por Amostra de } \\
\text { Domicílios (PNAD). }\end{array}$ & Positiva \\
\hline Integridade/ética & Direitos humanos: taxa de trabalho infantil (Trablnf). & Censo Demográfico 2010. & Negativa \\
\hline Conformidade legal & IRFS Fiscal (IFRS F). & CNM. & Positiva \\
\hline \multirow{3}{*}{ Equidade } & Índice de Gini (IndGini). & Censo Demográfico 2010. & Negativa \\
\hline & $\begin{array}{l}\text { Taxa de analfabetismo da população de } 15 \text { anos ou } \\
\text { mais (TxAnalf). }\end{array}$ & Censo Demográfico 2010. & Negativa \\
\hline & $\begin{array}{l}\text { Taxa de desemprego: percentual da população } \\
\text { de } 16 \text { anos ou mais, economicamente ativa, } \\
\text { desocupada (TxDes). }\end{array}$ & Censo Demográfico 2010. & Negativa \\
\hline \multirow{2}{*}{ Efetividade } & $\begin{array}{l}\text { Renda média domiciliar per capita percentual } \\
\text { (RendMDpc). }\end{array}$ & PNAD. & Positiva \\
\hline & $\begin{array}{l}\text { Índice de desenvolvimento humano municipal } \\
\text { (IDHM). }\end{array}$ & $\begin{array}{l}\text { Programa das Nações Unidas para o } \\
\text { Desenvolvimento (PNUD) no Brasil. }\end{array}$ & Positiva \\
\hline
\end{tabular}

Fonte: Elaborado pelos autores.

Variáveis de controle foram inseridas no modelo: densidade demográfica (DenDem); percentual de população urbana (PopUrb); média de anos de estudo dos adultos com mais de 25 anos (MedAE); e percentual de pessoas em domicílios com abastecimento de água e esgotamento sanitário inadequados (ÁguaEsg).

No modelo apresentado na equação 3, o IEM é uma variável dependente e os indicadores de governança pública são variáveis de interesse do modelo:

$$
\begin{aligned}
& \text { IEM }_{i}=\alpha+\text { IRFS }_{i} \beta_{1}+\text { ElVElA }_{i} \beta_{2}+\text { TxCM } \beta_{i}+\operatorname{PresCME}_{i} \beta_{4}+\operatorname{PresCMS}_{i} \beta_{5}+
\end{aligned}
$$

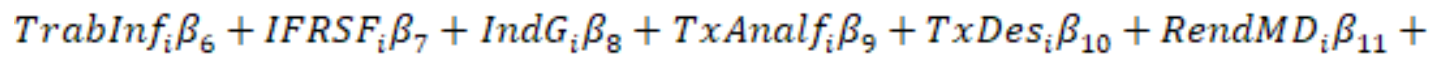

$$
\begin{aligned}
& \text { IDHM }_{i} \beta_{12}+\text { DenDem }_{i} \beta_{13}+\text { PopUrb }_{i} \beta_{14}+\text { MedAE }_{i} \beta_{15}+\% \text { ÁguaEsg } \beta_{i 6}+\varepsilon
\end{aligned}
$$

onde $\alpha$ é a constante da equação, $\beta_{\mathrm{n}}$ é o coeficiente que multiplica a variável $n$ da função e $\varepsilon$ é o termo de erro da equação.

Utilizou-se o softwate Stata ${ }^{\circledR}$, versão 12.0, no que diz respeito à estimação dos modelos de regressão propostos. 


\section{ANÁLISE E INTERPRETAÇÃO DOS RESULTADOS}

A Tabela 1 apresenta o valor do fator de inflação da variância (variance inflation factor - VIF) para todas as variáveis independentes do modelo.

\section{TABELA 1 FATOR DE INFLAÇÃO DE VARIÂNCIA DAS VARIÁVEIS DO MODELO}

\begin{tabular}{lc} 
Variável & Fator de inflação da variancia \\
IDHM & 16,36 \\
RendMDpc & 8,56 \\
TxAnalf & 6,48 \\
PopUrb & 2,53 \\
ÁguaEsg & 2,45 \\
IRFS & 2,00 \\
TxParCM & 1,97 \\
IRFSF & 1,78 \\
MedAE & 1,74 \\
TxDes & 1,61 \\
IndGini & 1,43 \\
EIVEIA & 1,38 \\
Trablnf & 1,37 \\
PresCME & 1,19 \\
DenDem & 1,14 \\
PresCMS & 1,06 \\
\hline
\end{tabular}

Fonte: Elaborada pelos autores.

Nota-se, pelo resultado da análise do VIF, que a variável Índice de desenvolvimento humano municipal (IDHM) apresentou um fator de inflação alto, o que pode trazer problema de multicolinearidade para o modelo (Fávero \& Belfiore, 2015). Desse modo, optou-se por retirar a variável IDHM dos modelos de regressão deste estudo. Todas as outras variáveis apresentaram VIF menor do que 10, assim, optou-se por mantê-las no modelo.

A Tabela 2 apresenta os resultados obtidos pelo modelo MQO e pela regressão Tobit bootstrapping. Optou-se por utilizar erros padrão robustos por conta do teste de heterocedasticidade de BreuschPagan apresentar uma estatística qui-quadrado de 38,30, com valor $p$ menor do que 0,01 . Ou seja, os resíduos do modelo são heterocedásticos, desse modo, assumem-se erros padrão robustos para corrigir esse problema. 


\section{TABELA 2 MODELO DE REGRESSÃO MQO E REGRESSÃO TOBIT BOOTSTRAPING IEM}

\begin{tabular}{|c|c|c|c|c|c|c|c|c|}
\hline \multirow{2}{*}{ Variáveis } & \multicolumn{4}{|c|}{ MQO } & \multicolumn{4}{|c|}{ Tobit (bootstrapping) } \\
\hline & Coeficiente & Erro padrão & Valor $p$ & Sig. & Coeficiente & Erro padrão & Valor $p$ & Sig \\
\hline EIV/EIA & $-0,00255$ & 0,00034 & 0,00 & \multirow[t]{2}{*}{ 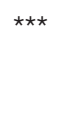 } & $-0,00255$ & 0,00033 & 0,00 & \multirow[t]{2}{*}{ *** } \\
\hline TxParCM & $-0,00013$ & 0,00016 & 0,42 & & $-0,00013$ & 0,00015 & 0,41 & \\
\hline PresCME & $-0,01371$ & 0,00445 & 0,00 & \multirow[t]{2}{*}{ 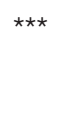 } & $-0,01371$ & 0,00433 & 0,00 & \multirow[t]{2}{*}{ 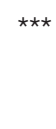 } \\
\hline PresCMS & 0,01123 & 0,01107 & 0,31 & & 0,01123 & 0,01084 & 0,30 & \\
\hline IRFS & 0,21299 & 0,05449 & 0,00 & \multirow[t]{2}{*}{ 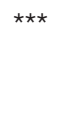 } & 0,21299 & 0,05410 & 0,00 & \multirow[t]{2}{*}{ *** } \\
\hline IRFSF & $-0,04094$ & 0,03837 & 0,29 & & $-0,04094$ & 0,03750 & 0,28 & \\
\hline Trablnf & $-0,00083$ & 0,00031 & 0,01 & 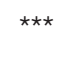 & $-0,00083$ & 0,00031 & 0,01 & *** \\
\hline IndGini & $-0,16284$ & 0,03315 & 0,00 & \multirow[t]{2}{*}{ 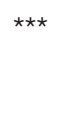 } & $-0,16284$ & 0,03278 & 0,00 & \multirow[t]{2}{*}{ *** } \\
\hline TxAnalf & 0,00043 & 0,00037 & 0,24 & & 0,00043 & 0,00036 & 0,23 & \\
\hline TxDes & $-0,00433$ & 0,00063 & 0,00 & 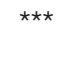 & $-0,00433$ & 0,00063 & 0,00 & *** \\
\hline RendMDpc & $-0,00012$ & 0,00002 & 0,00 & \multirow[t]{2}{*}{ 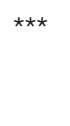 } & $-0,00012$ & 0,00002 & 0,00 & \multirow[t]{2}{*}{$* * *$} \\
\hline DenDem & 0,00000 & 0,00000 & 0,96 & & 0,00000 & 0,00000 & 0,96 & \\
\hline MedAE & 0,02289 & 0,00213 & 0,00 & 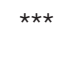 & 0,02289 & 0,00209 & 0,00 & $* * \star$ \\
\hline ÁguaEsg & 0,00063 & 0,00011 & 0,00 & \multirow[t]{2}{*}{ 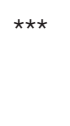 } & 0,00063 & 0,00011 & 0,00 & \multirow[t]{2}{*}{ *** } \\
\hline PopUrb & $-0,01025$ & 0,01239 & 0,41 & & $-0,01025$ & 0,01294 & 0,43 & \\
\hline _cons & 0,33548 & 0,04848 & 0,00 & \multirow[t]{5}{*}{ *** } & 0,33548 & 0,04554 & 0,00 & \multirow[t]{5}{*}{ *** } \\
\hline $\mathrm{R}^{2}$ & 0,1988 & & & & Pseudo $\mathrm{R}^{2}$ & 0,1275 & & \\
\hline $\mathrm{F}$ & 55,82 & & & & Wald qui² & $1.512,74$ & & \\
\hline Prob $>F$ & 0,0000 & & & & Prob $>$ qui ${ }^{2}$ & 0,0000 & & \\
\hline Observações & 3.193 & & & & Log Likelihood & $3.129,0603$ & & \\
\hline Breusch-Pagan & \multicolumn{3}{|c|}{ qui² 38,30 (valor $p=0,0000$ ) } & & & & & \\
\hline \multirow[t]{2}{*}{ Jaque-Bera } & \multicolumn{3}{|c|}{ qui² 308,80 (valor $p=0,0000$ ) } & & & & & \\
\hline & \multicolumn{3}{|c|}{ Nota: ${ }^{* \star \star}$ significância de 1\% } & & & & & \\
\hline
\end{tabular}

Fonte: Elaborada pelos autores.

Quanto ao princípio de participação da governança pública, somente as variáveis percentual de eleitores: votos válidos versus eleitores aptos - percentual no $1^{\circ}$ turno (ElV/ElA\%) e a presença de conselho municipal de educação (PresCME) foram estatisticamente significativas, no nível de confiança de 99\%. Tal resultado foi o inverso do esperado e diverge dos resultados obtidos por Asatryan e Witte (2015) e J. L. M. Silva e Almeida (2012). Costa et al. (2015) mostraram que, em anos de eleição, a eficiência da aplicação dos recursos públicos diminui por conta dos gestores aumentarem seus gastos visando a atender às demandas de certa parcela da população. Tal fenômeno pode repetir-se em municípios que apresentam maior presença de sua população nas eleições. 
Quanto ao princípio de transparência e accountability, a IRFS apresentou uma relação positiva com o IEM. Tal relação foi estatisticamente significativa no nível de $1 \%$ e confirma a relação esperada. O resultado corrobora os apresentados por C. F. Cruz, Ferreira, Silva e Macedo (2012). Municípios que apresentaram maior transparência de sua gestão, bem como melhores índices de gestão, foram os que obtiveram melhor eficiência na aplicação dos recursos públicos em educação e saúde.

Em relação ao princípio de integridade/ética, a variável TrabInf apresentou uma relação negativa com o IEM da aplicação dos recursos públicos em educação e saúde dos municípios brasileiros, convergindo com o resultado esperado e corroborando Hauner (2008). Quando a criança trabalha, presume-se que não está inserida na escola, o que pode ter reduzido o nível de atendimento dos municípios que apresentaram maior taxa de trabalho infantil.

No que tange ao princípio de governança pública na conformidade legal, a variável IRFS Fiscal apresentou uma relação negativa com o IEM da aplicação dos recursos públicos em educação e saúde. Porém, o efeito de tal variável não foi estatisticamente significativa.

Quanto ao princípio de governança pública de equidade, somente as variáveis IndGini e TxDes apresentaram relação negativa com o IEM da aplicação dos recursos públicos em educação e saúde. Assim, pode-se concluir que, no nível de significância de 1\%, a desigualdade social afeta de modo negativo a eficiência na aplicação dos recursos públicos, bem como o nível de desemprego. O resultado corrobora Hauner (2008) e Lopes e Toyoshima (2008).

No que diz respeito ao princípio de efetividade, a variável RendMDpc apresentou relação negativa com o IEM da aplicação dos recursos públicos em educação e saúde. Tal resultado foi o inverso do esperado e diverge dos resultados obtidos por Lopes e Toyoshima (2008). Tal resultado é estatisticamente significativo no nível de confiança de $99 \%$. Pode-se presumir que o aumento da renda faz com que aumente a busca por serviços privados de educação e saúde.

Por fim, dentre as variáveis de controle selecionadas para os modelos de regressão, somente as variáveis MedAE e ÁguaEsg foram estatisticamente significativas, ambas no nível de confiança de 99\%. A MedAE apresentou relação positiva com o IEM, resultado este em consonância com o resultado esperado e com os obtidos por Lopes e Toyoshima (2008) e K. E. A. Cruz e Ramos (2012). Percebe-se os benefícios das externalidades dos investimentos em educação, onde o incremento do capital humano afeta de forma positiva, não só no crescimento econômico (Zoghbi, Matos, Rocha, \& Arvate, 2009), mas também na eficiência da aplicação dos recursos públicos. Já ÁguaEsg apresentou relação positiva com o IEM da aplicação dos recursos públicos em educação e saúde, divergindo do resultado esperado.

De acordo com $\mathrm{R}^{2}$ do modelo de regressão $\mathrm{MQO}$, as variáveis que compuseram o modelo explicam 19,88\% das variações do IEM construído para esta pesquisa. Não houve normalidade dos resíduos para os modelos em questão. Entretanto, de acordo com o teorema do limite central e levando em consideração que a amostra foi composta por mais de 300 observações, relaxou-se o pressuposto da normalidade dos resíduos (Fávero \& Belfiore, 2015).

Os resultados da Tabela 3 ilustram o grau de associação das variáveis de governança pública com o IEM da aplicação dos recursos públicos em educação e saúde nos diferentes quintis aqui determinados. 


\section{TABELA 3 MODELO DE REGRESSÃO QUANTÍLICA IEM}

\begin{tabular}{|c|c|c|c|c|c|c|c|c|c|c|}
\hline \multirow{3}{*}{$\begin{array}{l}\text { Variáveis } \\
\text { EIV/EIA }\end{array}$} & \multicolumn{10}{|c|}{ Coeficientes } \\
\hline & \multicolumn{2}{|l|}{ q.10 } & \multicolumn{2}{|l|}{ q.25 } & \multicolumn{2}{|l|}{ q.50 } & \multicolumn{2}{|c|}{ q.75 } & \multicolumn{2}{|c|}{ q. .90} \\
\hline & $-0,00168$ & 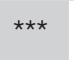 & $-0,00242$ & 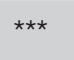 & $-0,00229$ & 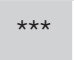 & $-0,00239$ & 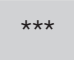 & $-0,00361$ & *** \\
\hline TxParCM & 0,00050 & $* * \star$ & 0,00001 & & $-0,00016$ & & $-0,00033$ & & $-0,00052$ & \\
\hline PresCME & $-0,01279$ & ** & $-0,01087$ & $* *$ & $-0,01318$ & $\star *$ & $-0,01400$ & * & $-0,01794$ & \\
\hline PresCMS & 0,00182 & & 0,02249 & * & 0,00626 & & 0,00361 & & 0,0009 & \\
\hline IRFS & 0,21595 & 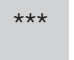 & 0,16770 & 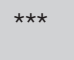 & 0,18682 & $\star \star \star ~$ & 0,28113 & $\star \star \star ~$ & 0,40037 & 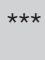 \\
\hline IRFSF & $-0,08834$ & & $-0,05739$ & & $-0,03799$ & & 0,03727 & & $-0,03717$ & \\
\hline Trablnf & 0,00064 & & $-0,00016$ & & $-0,00102$ & $* \star \star$ & $-0,00129$ & $\star \star \star ~$ & $-0,00177$ & ** \\
\hline IndGini & $-0,14820$ & 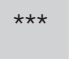 & $-0,19376$ & 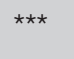 & $-0,17018$ & 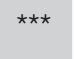 & $-0,09800$ & * & $-0,19237$ & ** \\
\hline TxAnalf & 0,00323 & & 0,00170 & & 0,00004 & & $-0,00103$ & * & $-0,00148$ & * \\
\hline TxDes & $-0,00524$ & 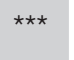 & $-0,00455$ & 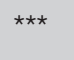 & $-0,00438$ & $* \star \star$ & $-0,00437$ & $\star \star \star ~$ & $-0,00386$ & *** \\
\hline RendMDpc & $-0,00004$ & $\star *$ & $-0,00007$ & $* \star \star$ & $-0,00012$ & $\star \star \star \star ~$ & $-0,00018$ & 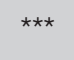 & $-0,00015$ & 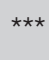 \\
\hline DenDem & 0,00000 & * & 0,00000 & & $-0,00000$ & & 0,00000 & & 0,00000 & \\
\hline MedAE & 0,01937 & 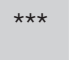 & 0,01837 & 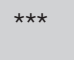 & 0,01992 & $* \star \star$ & 0,02569 & 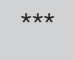 & 0,02606 & *** \\
\hline ÁguaEsg & 0,00029 & $\star \star$ & 0,00058 & $\star \star \star ~$ & 0,00068 & & 0,00059 & $\star \star \star ~$ & 0,00090 & 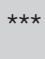 \\
\hline PopUrb & 0,04333 & $\star \star$ & 0,02522 & & $-0,00164$ & & $-0,03100$ & & $-0,05676$ & \\
\hline _cons & 0,06998 & & 0,25941 & 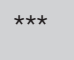 & 0,35912 & 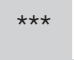 & 0,33705 & 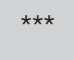 & 0,54125 & 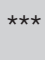 \\
\hline \multicolumn{11}{|c|}{ Nota: * significância de $10 \%$; ** significância de $5 \%$; ${ }^{* \star *}$ significância de $1 \%$} \\
\hline Pseudo $R^{2} .10$ & 0,1195 & & & & & & & & & \\
\hline Pseudo $R^{2} .25$ & 0,1168 & & & & & & & & & \\
\hline Pseudo $R^{2} .50$ & 0,1151 & & & & & & & & & \\
\hline Pseudo $R^{2} .75$ & 0,1129 & & & & & & & & & \\
\hline Pseudo $R^{2} .90$ & 0,1178 & & & & & & & & & \\
\hline \multicolumn{11}{|c|}{ Teste de Wald: $F=28,57 ;$ Prob $>F=0,0000$} \\
\hline Jaque-Bera: qu & $=455,00($ & alor $p$ & $=0,0000)$ & & & & & & & \\
\hline
\end{tabular}

Fonte: Elaborada pelos autores.

De modo geral, o resultado da regressão quantílica e dos outros modelos de regressão não divergem entre si, porém, podem-se verificar certas diferenças quando observardos os quintis individualmente.

No que diz respeito às variáveis do princípio de participação, a variável ElV/ElA apresentou o mesmo resultado para todos os quintis e repetindo a relação negativa encontrada nos outros modelos de regressão, ou seja, municípios que apresentaram maior participação nas eleições municipais apresentaram menores eficiências da aplicação dos recursos públicos com educação e saúde. Quanto à 
TxParCM, os municípios com os menores IEM (q.10) tiveram uma relação positiva com essa variável, ou seja, o número de conselhos municipais afeta positivamente até certo nível de eficiência da aplicação dos recursos públicos. Essa afirmação pode ser feita no nível de significância de $1 \%$. O resultado para essa variável é o mesmo que o esperado e corrobora os resultados de J. L. M. Silva e Almeida (2012) e Costa et al. (2015), porém, só foi observada para o menor quintil da amostra desta pesquisa.

A PresCME afetou de forma negativa a eficiência da aplicação dos recursos públicos em educação e saúde. Todos os modelos demonstraram essa relação negativa, com excessão do q.90 da regressão quantílica onde essa relação não é estatisticamente significativa. Costa et al. (2015) também demonstraram uma relação negativa entre o IEM e a presença de conselhos municipais de educação. Já a PresCMS só foi estatisticamente significativa com os municípios que compuseram o q.25. Essa relação foi positiva no nível de significância de 10\%, e converge com o resultado esperado à essa variável.

Da mesma maneira que os outros modelos, a variável IRFSF não apresentou relação estatisticamente significativa com nenhum dos quintis da regressão quantílica.

No que tange à variável TrabInf somente nos quantis mais altos foi verificada uma relação negativa estatisticamente significativa (q.50 e q.75 a 1\% de significância e q.90 a 5\% de significância). O resultado está em consonância com os resultados esperados e corrobora os resultados de Hauner (2008).

A variável IndGini repetiu os resultados encontrados nos modelos anteriores. Tal resultado reforça que a desigualdade social prejudica a eficiência da aplicação dos recursos públicos. Quanto à TxAnalf, nos modelos de MQO e regressão Tobit bootstrapping, não apresentaram relação estatisticamente significativa. Já no modelo de regressão quantílica, essa relação negativa foi estatisticamente significativa nos q.75 e q.90. Já a TxDes repete o resultado apresentado com os modelos anteriores.

A RendMDpc, variável utilizada para medir o princípio de governança pública da efetividade, também repetiu os resultados para todos os modelos de regressão utilizados. Pressupõe-se que o aumento da renda faz com que a busca pelos serviços privados de educação e saúde aumentem, o que resulta na diminuição da demanda dos serviços públicos. Nesta pesquisa, a diminuição da demanda afeta diretamente a eficiência da aplicação dos recursos públicos em educação e saúde, já que as taxas de atendimentos educacionais e o número de pessoas atingidas pelos programas públicos de saúde compõem os modelos DEA construídos para esta pesquisa.

A DenDem apresentou uma relação positiva estatisticamente significativa somente para os municípios que compuseram o q.10. Pode-se afirmar que, no nível de significância de 10\%, a densidade demográfica influencia de maneira positiva o IEM da aplicação dos recursos públicos em educação e saúde até certo nível de eficiência. Isso pode acontecer em função de maior densidade populacional representar maior demanda por educação e saúde sem que haja necessidade de maior alocação de recursos (J. L. M. Silva \& Almeida, 2012). Em J. L. M. Silva e Almeida (2012), Varela et al. (2012) e Costa et al. (2015) essa relação é inversa. Já o resultado encontrado converge com os obtidos por Lopes e Toyoshima (2008).

A MedAE apresentou uma relação positiva com o IEM da aplicação dos recursos públicos em educação e saúde para todos os quintis, no nível de significância de 1\%. Esse resultado corrobora os obtidos anteriormente. Da mesma maneira, a variável ÁguaEsg repetiu os resultados encontrados pelos modelos anteriores.

Por fim, a variável percentual de população urbana apresentou uma relação positiva somente com o q.10, no nível de significância de 5\%. Ou seja, municípios com menores IEM obtiveram melhora 
dessa eficiência em relação ao seu nível de urbanização. Costa et al. (2015) também encontram essa relação para os municípios com menor eficiência de seus modelos.

De acordo com o teste de Wald pode-se afirmar, no nível de significância de 1\%, que os coeficientes de inclinação de cada quantil são diferentes para todas as variáveis explicativas. Desse modo, a regressão quantílica é adequada para representar a relação entre as variáveis explicativas e o IEM. O teste Jaque-Bera apresentou uma estatística qui-quadrado de 455 com valor $p$ menor que 0,01 . Assim, não se pode aceitar a hipótese nula da normalidade dos resíduos. Porém, levando em consideração o teorema do limite central e que a amostra é composta por mais de 300 observações para cada quantil, relaxou-se o pressuposto da distribuição normal dos resíduos (Fávero \& Belfiore, 2015).

\section{CONCLUSÃO}

Este estudo teve por objetivo verificar a influência de práticas de governança pública na eficiência da aplicação dos recursos públicos em educação e saúde dos municípios brasileiros. Para tanto, fez-se uso de dois modelos de DEA, com o intuito de mensurar a eficiência da aplicação dos recursos públicos em cada uma dessas funções e a construção do IEM. Posteriormente, verificou-se a influência de práticas de governança pública no IEM construído.

A variável IRFS, selecionada para mensurar o princípio de transparência e accountability, apresentou uma relação positiva, estatisticamente significativa, com todos os modelos de regressão utilizados. O resultado é o esperado para essa variável e corrobora C. F. Cruz et al. (2012). Assim, pode-se concluir que transparência e accountability, bem como melhores índices de gestão, influenciam de modo positivo a eficiência da aplicação dos recursos públicos em educação e saúde.

A variável ElV/ElA apresentou uma relação negativa estatisticamente significativa em todos os modelos de regressão utilizados por esta pesquisa. Tal resultado é o inverso daquele esperado para essa relação e diverge dos resultados obtidos por Asatryan e Witte (2015). Porém, Costa et al. (2015) evidenciam que a eficiência da aplicação dos recursos públicos tende a diminuir em anos de eleição, quando os gestores acabam por fazer escolhas públicas que beneficiam apenas um setor da população. Tal fenômeno pode estar sendo observado em municípios que tendem a ter maior participação da população nas eleições municipais.

Para TxParCM se esperava uma relação positiva com a eficiência da aplicação dos recursos públicos em educação e saúde. Essa relação só foi comprovada para os municípios com menores IEM na regressão quantílica. Indo nesse sentido, a PresCME influencia de maneira negativa o IEM. A PresCMS só influenciou de maneira positiva o IEM em municípios do q.25. Logo, os resultados apresentados pelas variáveis utilizadas para mensurar o princípio de governança pública de participação não foram ao encontro do esperado e divergem dos resultados apresentados por J. L. M. Silva e Almeida (2012), Asatryan e Witte (2015) e Costa et al. (2015).

A variável TxTrabInf, apresentou relação negativa em todos os modelos de regressão estudados nesta pesquisa. O resultado apresentado vai ao encontro do esperado e corrobora os resultados de Hauner (2008). Assim, é possível afirmar que municípios que trabalham no sentido de mitigar o trabalho infantil apresentam maior eficiência na aplicação de seus recursos públicos em educação e saúde. Tendo em vista o resultado descrito, pode-se afirmar, então, que a governança pública influencia de maneira positiva a eficiência da aplicação dos recursos públicos em educação e saúde. 
As variáveis IndGini e TxDes, utilizadas para mensurar o princípio da equidade, mostraram influênciar de modo negativo o IEM para todos os modelos de regressão utilizados. Essa relação foi estatisticamente significativa e seu resultado vai ao encontro do esperado. Já a TxAnalf só apresentou relação estatisticamente significativa nos quintis q.75 e q.90 da regressão quantílica, confirmando o esperado. A busca por equidade mostrou influenciar a eficiência da aplicação dos recursos públicos em educação e saúde.

Por fim, quanto ao princípio de governança pública da efetividade, a variável RendMDpc apresentou uma relação negativa com o IEM. Tal resultado diverge do esperado e da literatura. $\mathrm{O}$ resultado encontrado pode estar associado a busca de serviços privados de educação quando há aumento da renda, o que pode afetar diretamente a taxa de atendimento do setor educação.

Tendo em vista os resultados apresentados, esta pesquisa atingiu seu objetivo, uma vez que demonstra a influência de práticas de governança pública na eficiência da alocação dos recursos públicos em educação e saúde. Como contribuição para a literatura, demonstra-se como é a relação de cada princípio de governança pública com a eficiência da aplicação dos recursos públicos em educação e saúde, podendo auxiliar em futuras tomadas de decisão. Vale destacar que, de acordo com a conceitualização de governança pública exposta na seção 2 deste artigo, percebe-se a importância da inclusão de diversos atores em redes no processo de gestão pública (Dias \& Cario, 2014b; Kickert, 1997; Lynn \& Malinowska, 2018; Pedersen \& Johannsen, 2018) e a participação deles como agentes ativos, principalmente a inclusão da sociedade civil nesses processos. Este artigo mostrou que o efeito do princípio da participação apresentou resultado inverso ao esperado segundo a literatura. Costa et al. (2015) discutem que, em anos de eleição, a eficiência da aplicação dos recursos públicos diminui - como discutido. Quanto a presença de conselhos municipais influenciou de modo negativo ou não afetou a eficiência da aplicação dos recursos públicos, levanta-se a dúvida sobre o fato da presença e participação da sociedade civil em tais conselhos ter-se dado de forma efetiva para a amostra estudada e como se desenha essa participação nos conselhos.

No que tange às futuras pesquisas, destaca-se que o tema governança pública e sua relação com o desempenho da máquina pública é pouco explorado academicamente em nível nacional. Assim, várias possibilidades de pesquisa se apresentam a partir dos resultados deste estudo, como, por exemplo, entender o sistema eleitoral brasileiro com o desempenho da aplicação dos recursos públicos e suas escolhas públicas, já que a variável utilizada para mensurar a participação nas eleições municipais mostrou influenciar de modo negativo a eficiência da aplicação dos recursos públicos em educação e saúde. Como se dá a participação da sociedade civil na tomada de decisão dentro dos conselhos municipais, entre outras possibilidades.

Espera-se que esta pesquisa estimule os gestores públicos a buscar ações continuadas que aumentem a eficiência da aplicação de seus recursos, a fim de contribuir com o desenvolvimento socioeconômico. Destaca-se, ainda, a importância da governança pública para promover a eficiência da máquina pública e a relevância desta última no crescimento do estado, no sentido da redução do desperdício dos recursos públicos e da promoção da melhoria da qualidade de vida e do bem-estar da sociedade como um todo.

Vale ressaltar, no entanto, que os resultados desta pesquisa devem ser interpretados com cautela quanto à sua generalização, já que se limitam à amostra investigada e podem mostrar-se sensíveis às decisões metodológicas tomadas para a realização deste estudo. 


\section{REFERÊNCIAS}

Adkins, L. C. (2014). Using gretl for principles of econometrics. Stillwater, OK: Oklahoma State University.

Afonso, A., Schuknecht, L., \& Tanzi, V. (2010). Public sector efficiency: evidence for new $\mathrm{EU}$ member states and emerging markets. Applied Economics, 42(17), 2147-2164.

Araújo, C. H., Conde, F. N., \& Luzio, N. (2007). Índice de qualidade da educação fundamental (IQE): proposta para discussão. Revista Brasileira de Estudos Pedagógicos, 85(209), 10-11.

Araújo-Júnior, J. N., Justo, W. R., Rocha, R. M., \& Gomes, S. M. F. P. O. (2017). Eficiência técnica das escolas públicas dos estados do Nordeste: uma abordagem em dois estágios. Revista Econômica do Nordeste, 47(3), 61-73.

Aristóteles. (2002). Ética a Nicômaco (5a ed.). São Paulo, SP: Martin Claret.

Asatryan, Z., \& Witte, K. (2015). Direct democracy and local government efficiency. European Journal of Political Economy, 39, 58-66.

Baciu, L., \& Botezat, A. (2014). A comparative analysis of the public spending efficiency of the new EU member states: a DEA approach. Emerging Markets Finance and Trade, 50(sup4), 31-46.

Banker, R. D., Charnes, A., \& Cooper, W. W. (1984). Some models for estimating technical and scale inefficiencies in data envelopment analysis. Management Science, 30(9), 1078-1092.

Chan, S.-G., \& Karim, M. Z. A. (2012). Public spending efficiency and political and economic factors: evidence from selected East Asian countries. Economic Annals, 57(193), 7-23.

Chang, M.-C. (2014). Efficiency and governance of power corporations: a China and Taiwan analysis. Polish Journal of Environmental Studies, 23(5), 1515-1525.

Considine, M., \& Lewis, J. M. (2003). Bureaucracy, network, or enterprise? Comparing models of governance in Australia, Britain, the Netherlands, and New Zealand. Public Administration Review, 63(2), 131-140.

Costa, C. C. M., Ferreira, M. A. M., Braga, M. J., \& Abrantes, L. A. (2015). Fatores associados à eficiência na alocação de recursos públicos à luz do modelo de regressão quantílica. Revista de Administração Pública, 49(5), 1319-1347.

Cruz, C. F., Ferreira, A. C. S., Silva, L. M., \& Macedo, M. A. S. (2012). Transparência da gestão pública municipal: um estudo a partir dos portais eletrônicos dos maiores municípios brasileiros. Revista de Administração Pública, 46(1), 153-76.

Cruz, K. E. A., \& Ramos, F. S. (2012). Eficiência na gestão do saneamento básico e seus impactos sobre a promoção da saúde: uma aplicação da análise envoltória de dados - DEA. In Anais do 17 E Encontro Regional de Economia. Fortaleza, CE.

Dias, T., \& Cario, S. A. F. (2014a). Sociedade, desenvolvimento e o papel estratégico do Estado: uma reflexão sobre o surgimento da governança pública. Ensaios FEE, 35(2), 337-362.

Dias, T., \& Cario, S. A. F. (2014b). Governança pública: ensaiando uma concepção. Contabilidade, Gestão e Governança, 17(3), 89-108.

Diniz, J. A. (2012). Eficiência das transferências intergovernamentais para a educação fundamental de municípios brasileiros (Tese de Doutorado). Universidade de São Paulo, São Paulo, SP.

European Comission (2001). European governance - a white paper. Recuperado em 10 de dezembro, 2016, de http://europa.eu/rapid/press-release DOC-01-10_en.htm.

Faria, F. P., Jannuzzi, P. M., \& Silva, S. J. (2008). Eficiência dos gastos municipais em saúde e educação: uma investigação através da análise envoltória no Estado do Rio de Janeiro. Revista de Administração Pública, 42(1), 155-177.

Fávero, L. P., \& Belfiore, P, F. (2015). Análise de dados: técnicas multivariadas exploratórias com SPSS ${ }^{\circ}$ Stata ${ }^{\circ}$. Rio de Janeiro, RJ: Elsevier.

Guerreiro Ramos, A. (1989). A nova ciência das organizações. Rio de Janeiro, RJ: Ed. FGV.

Hauner, D. (2008). Explaining differences in public sector efficiency: evidence from Russia’s regions. World Development, 36(10), 1745-1765.

Hoff, A. (2007). Second stage DEA: comparison of approaches for modelling the DEA score. European Journal of Operational Research, 181(1), 425-435. 
Hood, C. (1991). A public management for all seasons? Public Administration, 69(1), 3-19.

International Federation of Accountants. (2001). Study 13: governance in the public sector: a governing body perspective. New York, NY: Author.

Kickert, W. J. M. (1997). Public governance in the Netherlands: an alternative to Anglo-American 'managerialism'. Public Administration, 75(4), 731752.

Kissler, L., \& Heidemann, F. G. (2006). Governança pública: novo modelo regulatório para as relações entre Estado, mercado e sociedade? Revista de Administração Pública, 40(3), 479-499.

Kormendi, R. C., \& Meguire, P. G. (1985). Macroeconomic determinants of growth: crosscountry evidence. Journal of Monetary Economics, 16(2), 141-163.

Lampe, H. W., \& Hilgers, D. (2015). Trajectories of efficiency measurement: a bibliometric analysis of DEA and SFA. European Journal of Operational Research, 240(1), 1-21.

Lopes, L. S., \& Toyoshima, S. H. (2008). Eficiência técnica municipal na gestão dos gastos com saúde e educação em Minas Gerais: seus impactos e determinantes. In Anais do 130 Seminário sobre a Economia Mineira. Diamantina, MG.

Lopes, L. S., Toyoshima, S. H., \& Gomes, A. P. (2009). Determinantes sociais da saúde em Minas Gerais: uma abordagem empírica. In Anais do 7o Encontro Nacional da Associação Brasileira de Estudos Regionais e Urbanos. São Paulo, SP.

Lynn, L. E., Jr., \& Malinowska, A. (2018). How are patterns of public governance changing in the US and the EU? It's complicated. Journal of Comparative Policy Analysis: Research and Practice, 20(1), 36-55.

Mandeli, K. N. (2016). New public governance in Saudi cities: an empirical assessment of the quality of the municipal system in Jeddah. Habitat International, 51, 114-123.

Matias-Pereira, J. (2010). A governança corporativa aplicada no setor público brasileiro. Administração Pública e Gestão Social, 2(1), 109-134.

Mello, J. C. C. B. S., Angulo-Meza, L., Gomes, E. G., Fernandes, A. J. S., \& Biondi, L., Neto. (2008). Estudo não paramétrico da relação entre consumo de energia, renda e temperatura. IEEE Latin America Transactions, 6(2), 153-161.

Oliveira, I. G. S. (2015). Análise de correspondência aplicada a proposições sobre a eficiência e eficácia do processo de compras públicas a partir da percepção de diferentes atores envolvidos. In Anales del 90 Congreso Iberoamericano de Contabilidade de Gestión. Florianópolis, SC.

Oliveira, A. G., \& Pisa, B. J. (2015). IGovP: índice de avaliação da governança pública - instrumento de planejamento do Estado e de controle social pelo cidadão. Revista de Administração Pública, 49(5), 1263-1290.

Organização das Nações Unidas para a Educação, a Ciência e a Cultura. (1998). Declaração Mundial sobre Educação para Todos. Recuperado de http://unesdoc. unesco.org/images/0008/000862/086291por.pdf

Organisation for Economic Co-Operation and Development. (2001). Citizens as partners: information, consultation and public participation in policy-making. Paris, France: Author.

Pedersen, K. H., \& Johannsen, L. (2018). New public governance in the Baltic States: flexible administration and rule bending. Public Performance \& Management Review, 41(3), 648-667.

Peña, C. R. (2008). Um modelo de avaliação da eficiência da administração pública através do método análise envoltória de dados (DEA). Revista de Administração Contemporânea, 12(1), 83-106.

Peters, B. G., \& Pierre, J. (1998). Governance without government? Rethinking public administration. Journal of Public Administration Research and Theory, 8(2), 223-243.

Pisa, B. J.(2014). Uma proposta para o desenvolvimento do indice de avaliação da governança pública (IGovP): instrumento de planejamento e desenvolvimento do Estado (Dissertação de Mestrado). Universidade Tecnológica Federal do Paraná, Curitiba, PR.

Secchi, L. (2009). Modelos organizacionais e reformas da administração pública. Revista de Administração Pública, 43(2), 347-369.

Samut, P. K., \& Cafrı, R. (2016). Analysis of the efficiency determinants of health systems in OECD countries by DEA and panel tobit. Social Indicators Research, 129(1), 113-132. 
Sibiano, P., \& Agasisti, T. (2013). Efficiency and heterogeneity of public spending in education among Italian regions. Journal of Public Affairs, 13(1), 12-22.

Silva, A. A. P., Ferreira, M. A. M., Braga, M. J., \& Abrantes, L. A. (2012). Eficiência na alocação de recursos públicos destinados à educação, saúde e habitação em municípios mineiros. Contabilidade, Gestão e Governança, 15(1), 96-114.

Silva, J. L. M., \& Almeida, J. C. L. (2012). Eficiência no gasto público com educação: uma análise dos municípios do Rio Grande do Norte. Planejamento e Políticas Públicas, n. 39, 219-242.

Simar, L. (2003). Detecting outliers in frontier models: a simple approach. Journal of Productivity Analysis, 20(3), 391-424.

Souza, C. V. N., Júnior, \& Gasparini, C. E. (2006). Análise da equidade e da eficiência dos estados no contexto do federalismo fiscal brasileiro. Estudos Econômicos, 36(4), 803-832.
Tribunal de Contas da União. (2014). Governança pública: referencial básico de governança aplicável a órgãos e entidades da administração pública e ações indutoras de melhoria. Brasília, DF: Secretaria de Planejamento, Governança e Gestão.

Varela, P. S., Martins, G. A., \& Fávero, L. P. L. (2012). Desempenho dos municípios paulistas: uma avaliação de eficiência da atenção básica à saúde. Revista de Administração (São Paulo), 47(4), 624-637.

World Bank. (2007). Governance and management. In Independent Evaluation Group, Sourcebook for evaluating global and regional partnership programs: indicative principles and standards (pp. 71-82). Washington, DC: Author.

Zoghbi, A. C. P., Matos, E. H. C., Rocha, F. F., \& Arvate, P. R. (2009). Mensurando o desempenho e a eficiência dos gastos estaduais em educação fundamental e média. Estudos Econômicos, 39(4), 785-809.

\section{Rodolfo Rocha dos Santos}

https://orcid.org/0000-0002-1511-6546

Mestre em Contabilidade pelo Programa de Pós-Graduação em Contabilidade da Universidade Federal de Santa Catarina (PPGC/UFSC); Professor Assistente no Departamento de Ciências Contábeis e Finanças da Universidade Federal Rural do Rio de Janeiro (DCCF/UFRRJ). E-mail: rodolforsantos@ufrrj.br

\section{Suliani Rover}

https://orcid.org/0000-0001-8612-2938

Doutora em Controladoria e Contabilidade pela Faculdade de Economia, Administração e Contabilidade da Universidade de São Paulo (FEA USP); Professora Adjunta no PPGC/UFSC. E-mail: sulianirover@gmail.com 